\title{
A METAPHYSEAL FRACTURE RAT MODEL FOR MECHANISTIC STUDIES OF OSTEOPOROTIC BONE HEALING
}

\author{
R.M.Y. Wong ${ }^{1}$, U. Thormann², M.H.V. Choy ${ }^{1}$, Y.N. Chim ${ }^{1}$, M.C.M. Li ${ }^{1}$, J.Y. Wang ${ }^{1}$, K.S. Leung ${ }^{1}$, \\ J.C.Y. Cheng ${ }^{1}$, V. Alt ${ }^{2,}$, S.K.H. Chow ${ }^{1, \S *}$ and W.H. Cheung ${ }^{1}$ \\ ${ }^{1}$ Department of Orthopaedics and Traumatology, Prince of Wales Hospital, \\ The Chinese University of Hong Kong, Hong Kong, The People's Republic of China \\ ${ }^{2}$ Department of Orthopaedic Trauma Surgery, Justus-Liebig-University Giessen, Germany \\ ${ }^{3}$ Department of Trauma Surgery, Regensburg University Medical Centre, Regensburg, Germany \\ $\S$ Those authors contributed equally
}

\begin{abstract}
Most osteoporotic fractures occur at metaphyseal regions of long bones. The present study proposed a clinically relevant animal model that satisfied: i) induction of osteoporosis, ii) unilateral complete osteotomy at metaphysis, iii) internal fixation. 6 months old female Sprague-Dawley rats $(n=64)$ were randomly divided into the ovariectomised-metaphyseal osteotomy (OVX, $n=32)$ and metaphyseal osteotomy (SHAM, $n=32)$ groups. The metaphyseal-osteotomy model was created with a plate-fixation of the osteotomy and assessed by X-ray, micro-computed tomography, histomorphometry and mechanical testing at weeks 1, 3 and 6. X-ray results showed complete healing of metaphyseal osteotomy at week 6 . Histology showed 3 stages of metaphyseal healing. Stage 1 was characterised by fibrous tissue, consisting of disorganised orientation of collagen fibres, and infiltration of immune cells. At stage 2, a transitional zone consisting of maturing fibrous tissue and differentiating mesenchymal cells with early trabecular bone formation and disorganised woven bone were observed. During stage 3, cortical bone ends unified and woven bone underwent transformation to lamellar bone. OVX group healing was significantly delayed when compared to SHAM samples.

The study demonstrated that healing of osteoporotic osteotomy at the metaphyseal region was delayed in terms of radiography, histomorphometry and mechanical strength. These quantitative evaluations, along with histological features, may provide key references for future studies. The animal model may provide additional clinical relevance as most osteoporotic fracture in humans occurs at metaphyseal regions.
\end{abstract}

Keywords: Osteoporotic fracture healing, metaphyseal fracture, intramembranous ossification, endochondral ossification.

*Address for correspondence: Simon Kwoon-Ho Chow, Department of Orthopaedics and Traumatology, Prince of Wales Hospital, The Chinese University of Hong Kong, Shatin, New Territories, Hong Kong, China. Telephone number: +852 35053312 Email: skhchow@ort.cuhk.edu.hk

Copyright policy: This article is distributed in accordance with Creative Commons Attribution Licence (http://creativecommons.org/licenses/by-sa/4.0/).

\section{Introduction}

Osteoporosis is a major skeletal disorder predisposing patients to increased risk of fragility fractures. Despite advances in the prevention and treatment of osteoporotic fractures, their prevalence continues to rise (Rachner et al., 2011). There are approximately 2.5 million osteoporotic fractures each year in the United States, resulting in increased morbidity, mortality and healthcare expenditures (Solomon et al., 2014). Failure of fracture union results in pain, weakness and reduced mobility and these complications are most common in elderly patients.
Most osteoporotic fractures occur at metaphyseal regions of long bones (Larsson, 2002), including distal radius, proximal humerus or proximal femur (Alt et al., 2013). Despite the evidence, most pre-clinical studies have concentrated on healing of osteoporotic diaphyseal fractures with intramedullary fixation based on the classic model described by Bonnarens and Einhorn (1984). Few experimental studies have been performed on osteoporotic metaphyseal osteotomies on rats (Kolios et al., 2010; Komrakova et al., 2015; Stuermer et al., 2013), goats (Cheung et al., 2016) and sheep (Bindl et al., 2013). Amongst these limited studies, many are clinically inadequate, with 
over-simplified models including drill-hole defects and partial osteotomy models (Wong et al., 2018). Within the remaining complete osteotomy models, most are performed on both limbs of the animal. However, bilateral osteotomies should be avoided due to the negative influence on the weight bearing status of the animal (Auer et al., 2007). Therefore, the use of a unilateral limb complete osteotomy is more appropriate. Currently, there is a lack of clinically relevant models comparing osteoporotic with normal bones; therefore, metaphyseal bone repair is poorly understood (Cheung et al., 2016; Histing et al., 2012; Wong et al., 2018).

A recently published systematic review recommended that a clinically relevant osteoporotic metaphyseal fracture animal model should satisfy the following criteria: i) induction of osteoporosis, ii) complete osteotomy or defect unilaterally, iii) internal fixation (Wong et al., 2018). The primary objective of the present study was to present a reproducible, clinically relevant animal model that satisfied the guidelines for future osteoporotic bone studies and clinical translation.

\section{Materials and Methods}

Experimental design and induction of osteoporosis 64 six-month old female Sprague-Dawley rats $(n=64)$, weighing 200-250 g, were obtained from the Laboratory Animal Services Centre of The Chinese University of Hong Kong, China (animal experimental ethics committee reference number: 16037-MIS). Following 1 week of acclimatisation (Leung et al., 2009), rats were randomly divided into 2 groups: ovariectomised metaphyseal osteotomy (OVX, $n=32)$ and SHAM metaphyseal osteotomy (SHAM, $n=32$ ). Under general anaesthesia (Shi et al., 2010) with intra-peritoneal injection of ketamine $(20-60 \mathrm{mg} / \mathrm{kg})$ and xylazine $(2.5 \mathrm{mg} / \mathrm{kg})$, the OVX group underwent bilateral ovariectomy, with a dorsolateral approach to both ovaries performed sequentially. The peritoneal cavity was entered and the ovary was identified, ligated and removed. SHAM rats underwent the same procedure but ovaries were identified and kept intact. $0.05 \mathrm{mg} / \mathrm{kg}$ intra-muscular buprenorphine was given 15 min before surgery and for 3 consecutive days after the procedure to minimise pain. The rats were housed for 3 months before fracture induction.

Rats were euthanised at $1(n=8), 3(n=8)$ and 6 weeks $(n=8)$. Assessments included clinical analysis, X-ray, micro-computed tomography $(\mu \mathrm{CT})$ and histomorphometry. The remaining 8 rats were also euthanised at week 6 for mechanical testing, which required a separate batch.

Bone mineral density (BMD) was assessed by dual energy X-ray absorptiometry (DXA; UltraFocus, Faxitron, Houston, TX, USA) at the lumbar spine, proximal femur and distal femur. Each rat was subjected to DXA while under general anaesthesia, pre-ovariectomy at 6 months and pre-fracture induction at 9 months.

\section{Metaphyseal osteotomy model}

After general anaesthesia with intra-peritoneal injection of ketamine $(20-60 \mathrm{mg} / \mathrm{kg})$ and xylazine $(2.5 \mathrm{mg} / \mathrm{kg})$, the left femur was disinfected with povidone-iodine [Betadine ${ }^{\circledast}$ Antiseptic topical solution 10 \%, Mundipharma (Hong Kong) Ltd., Hong Kong, China] and draped in sterile material. A $25 \mathrm{~mm}$-long incision was made along the lateral aspect of the distal femur. The fascia was incised. The vastus lateralis and lateral head of biceps femoris were split to access the lateral condyle to the femur mid-shaft. Joint capsule arthrotomy was performed and the patella was dislocated medially for a better exposure and to facilitate the subsequent osteotomy. A 6-hole $1.0 \mathrm{~mm}$-long T-shaped mini-plate (T-plates, Bioortho $^{\circledR}$, Suzhou, China) was used to fix the lateral femur with $1.1 \mathrm{~mm}$ self-tapping cortical screws. Two $8 \mathrm{~mm}$ screws were inserted perpendicular to the knee articular surface at the distal femur and three $6 \mathrm{~mm}$ screws proximally to the shaft. A small oscillating saw, $0.35 \mathrm{~mm}$ in width (OT7S-3, Piezosurgery ${ }^{\circledR}$ Touch, Metron ${ }^{\circledR}$, Carasco, Italy), was used to create an osteotomy just proximal to the epiphyseal cartilage at the metaphysis (Fig. 1). The wound was irrigated with saline. Then, the patella was reduced into position and the joint capsule arthrotomy was repaired with a 4-0 vicryl suture. The fascia was repaired with a 4-0 vicryl suture and the skin was closed with a 5-0 nylon suture. $0.05 \mathrm{mg} /$ $\mathrm{kg}$ intra-muscular buprenorphine was given $15 \mathrm{~min}$ before surgery and for 3 consecutive days after the procedure to minimise pain.

\section{Clinical monitoring}

Animal welfare was observed in terms of a score sheet based on the New Zealand "Good practice guide for the use of animals in research, testing and teaching" (Web Ref. 1). The observed parameters included body weight, balance of risk (BAR) score, general clinical signs, behavioural signs of pain, water balance, operation site and post-operative support. A post-operative care card from the Department of Orthopaedics and Traumatology, Prince of Wales Hospital, The Chinese University of Hong Kong, China was also used to document treatment.

\section{X-ray plain radiograph}

Healing was monitored by two independent surgeons, as previously described (Leung et al., 2009). Anteroposterior and lateral views were acquired. The exposure settings for each of the $\mathrm{X}$-ray image were $32.0 \mathrm{kV}, 0.34 \mathrm{~mA}, 1.73 \mathrm{mAs}, 5.03 \mathrm{~s}$ (UltraFocus DXA, Faxitron, AZ, USA). For the evaluation of the relative radio-opacity, each measurement of the osteotomy site was normalised to the radio-opacity sampled at the metal implant where X-ray attenuation was the highest. The metaphyseal osteotomy site was 
assessed through 3 randomly selected regions of $5 \times 5$ pixels each, whose intensity was quantified using the image analysis software ImageJ.

\section{Specimen harvest}

Metaphyseal-osteotomy rats were sacrificed at week 1,3 and 6 as healing was achieved at 6 weeks (Alt et al., 2013). 8 rats were assigned to each time point with a separate batch for mechanical testing at the end-point. Rats were administered with an overdose intra-peritoneal injection of sodium pentobarbital to induce euthanasia. The left femur was harvested and the implants were removed.

\section{$\mu \mathrm{CT}$ analysis}

Harvested femora $(n=8)$ were scanned by $\mu \mathrm{CT}(\mu \mathrm{CT}$ 40, Scanco Medical, Brüttisellen, Switzerland) at each time point according to an established protocol (Chow et al., 2011; Shi et al., 2010). A small region of interest (ROI), $1.8 \mathrm{~mm}$ above and below the osteotomy site, was selected to avoid the diaphyseal region. Microarchitectural parameters were evaluated in terms of tissue volume (TV), bone volume (BV), trabecular bone number (Tb.N), trabecular thickness (Tb. $\mathrm{Th})$, trabecular bone separation (Tb.Sp), trabecular number (Tb.N), trabecular spacing (Tb.Sp), apparent $\mathrm{BMD}$ at the TV [BMV(TV)] and BV [BMV(BV)], connectivity density (Conn-Dens) and structural model index (SMI).

\section{Histomorphometric analysis}

After $\mu \mathrm{CT}$, femora were decalcified, cut into two halves along mid-sagittal plane and embedded in paraffin wax. $5 \mu \mathrm{m}$-thick specimens were cut and stained with haematoxylin-eosin (H\&E) and safranin $\mathrm{O}(\mathrm{SO})$. Each specimen slide was examined and analysed by microscopy (Leica DMRB DAS). Three microscopic views per specimen for SHAM and OVX osteotomy sites captured, using a 40× objective, were randomly sampled for osteoblast (N.Ob) and osteoclast (N.Oc) number and analysed by histomorphometry (Osteomeasure ${ }^{\circledR}$, OsteoMetrics, Decatur, USA). Bone area (B.Ar) and tissue area (T.Ar) were evaluated covering $1.5 \mathrm{~mm}$ proximal and distal to the osteotomy site (total $3 \mathrm{~mm}$ ) (ImageJ). Safranin O uptake was evaluated for all groups (ImageJ).
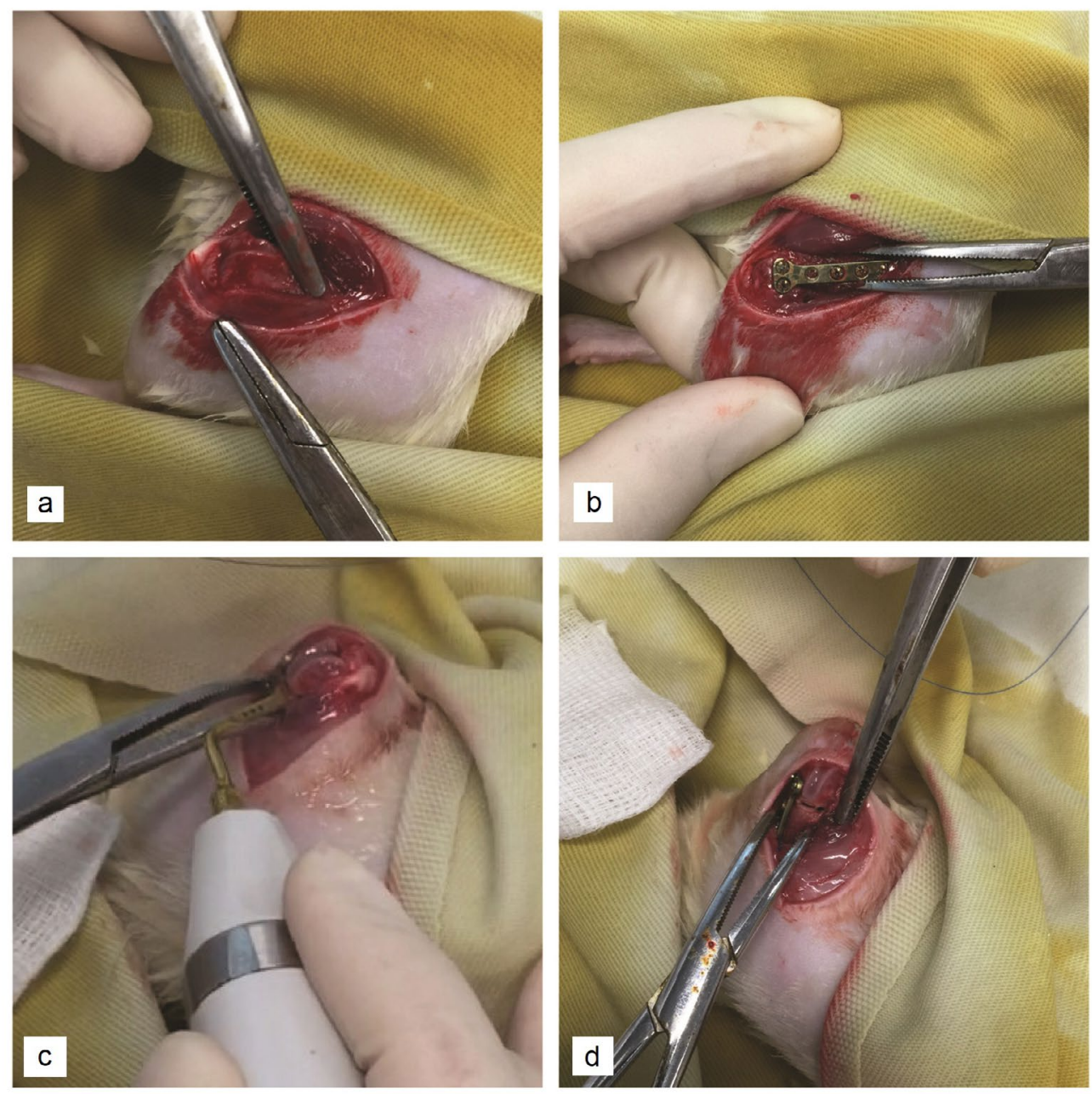

Fig. 1. Surgical technique for the metaphyseal osteotomy model. (a) An incision was made to expose the lateral condyle to the mid-shaft of the femur. (b) A 6-hole $1.0 \mathrm{~mm}$ T-shaped mini-plate was used to fix the lateral femur. (c) A small oscillating saw of $0.35 \mathrm{~mm}$ in width was used to create an osteotomy immediately proximal to the epiphyseal cartilage at the metaphyseal region. (d) The wound was irrigated with sterile saline and closed in layers using 4-0 vicryl and 5-0 nylon sutures. 


\section{Mechanical testing}

Mechanical testing for OVX, SHAM and contralateral intact bones was performed at week $6(n=8)$. Each femur was embedded in a performance polymer (Ureol 2020, Ciba, Hong Kong, China) (Leung et al., 2006) at the proximal end and fixed to the materialtesting machine (H25KS Hounsfield Test Equipment Ltd. Redhill, Surrey, UK) $25^{\circ}$ above the horizontal line, where the angle mimics the in situ physiological loading conditions (Fig. 2). A compressive force at the constant downward velocity of $25 \mathrm{~mm} / \mathrm{min}$ was applied by a metal blade at the epiphysis, directly above the second screw hole. After testing-tofailure, load-displacement curves were generated. Ultimate load $(\mathrm{N})$, stiffness $(\mathrm{N} / \mathrm{mm})$ and energy to failure $(\mathrm{N} \times \mathrm{mm})$ were recorded and analysed using a built-in software (QMAT Professional Material testing software, Hounsfield Test Equipment Ltd. Redhill, Surrey, UK) (Shi et al., 2010). Normalisation was calculated as OVX/OVX-intact-bone and SHAM/SHAM-intact-bone. Normalisation with the contralateral intact bone provided data in the relative mechanical strength of the bone after healing.

\section{Statistical analysis}

All quantitative data were expressed as mean \pm standard deviation and analysed with SPSS version 24.0 software (SPSS Inc., Chicago, IL, USA). Student $t$-test was used to compare BMD values. Two-way ANOVA and post-hoc test were used to compare differences at different time points. Significant

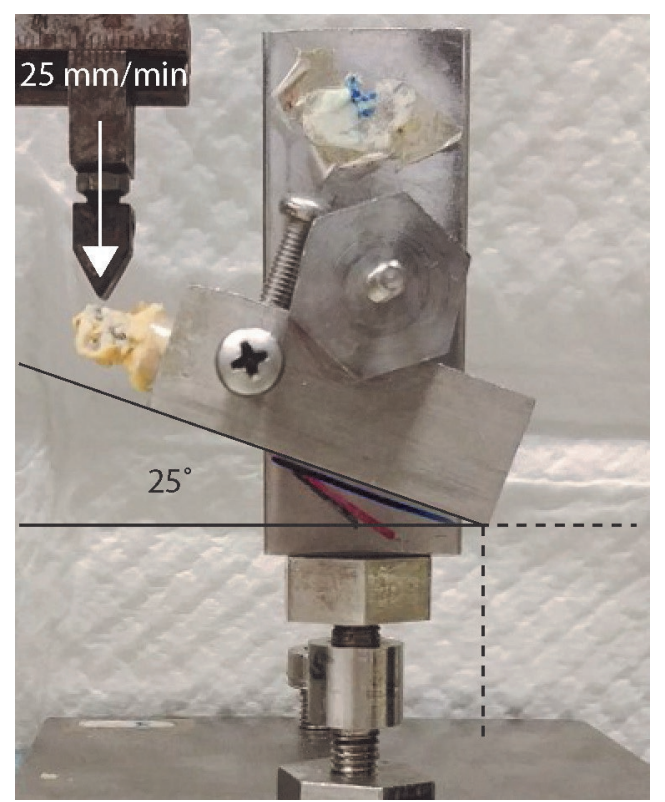

Fig. 2. Mechanical test of the metaphyseal fracture model. Each femur was embedded in a performance polymer at the proximal end and fixed to the material-testing machine $25^{\circ}$ above the horizontal line. The angle was chosen to mimic the in situ physiological loading conditions. A compressive force at a constant downward velocity of $25 \mathrm{~mm} / \mathrm{min}$ was applied by a metal blade at the epiphysis, directly above the second screw hole. difference was set at $p \leq 0.05$. Data normality was confirmed by Kolmogorov-Smirnov test.

\section{Results}

\section{Clinical results}

One rat died from general anaesthesia within the OVX group; the others were alert and responsive. 31 OVX (97\%) and 32 SHAM (100 \%) rats survived throughout the entire observation period. All rats resumed weight-bearing after surgery and no weight loss of $10 \%$ or more was measured throughout the study. No clinical complications, wound complications or behavioural signs of pain were observed. Rats were placed 4 per cage and drank an amount of water corresponding to around $10 \%$ of their body weight per day.

All rats achieved healing at the metaphysis. No evidence of screw hole loosening was present at sacrifice.

\section{Osteoporotic induction}

Mean BMD over L3-L5 lumbar spine decreased from $309 \pm 30.6 \mathrm{mg} / \mathrm{cm}^{2}$ to $267.9 \pm 14.8 \mathrm{mg} / \mathrm{cm}^{2}(p=0.05)$, over proximal femur from $428.1 \pm 5.10 \mathrm{mg} / \mathrm{cm}^{2}$ to $293.4 \pm 1.97 \mathrm{mg} / \mathrm{cm}^{2}(p<0.001)$ and over distal femur from $396.0 \pm 1.79 \mathrm{mg} / \mathrm{cm}^{2}$ to $309.1 \pm 3.50 \mathrm{mg} / \mathrm{cm}^{2}$ $(p<0.001)$.

\section{X-ray plain radiograph}

Complete healing occurred at 6 weeks for metaphyseal osteotomies. No significant callus formation was observed (Fig. 3a,b). Radiopacity of OVX osteotomy site was increased at week 6 as compared to week 1 $(p=0.002)$ and $2(p=0.007)$. Radiopacity of SHAM osteotomy site was increased at week 6 as compared to week $1(p=0.047)$. Radiopacity of SHAM group was significantly more at week 6 as compared to OVX group ( $p=0.045)$ (Fig. 4).

\section{$\mu \mathrm{CT}$}

$\mu \mathrm{CT}$ showed complete osteotomy performed at metaphyseal region with evidence of cancellous trabecular bone at proximal and distal ends (Fig. 3c).

Quantitative analysis of OVX rats demonstrated an increase in total BV from week 1 to week 3 $(p=0.00)$ and from week 3 to week $6(p=0.00)$. Total TV increased between week 1 and week 3 and 6 $(p=0.00) . \mathrm{BV} / \mathrm{TV}$ increased between week 1 and 3 as compared to week $6(p=0.009$ and $p=0.006$, respectively). Tb.N was larger from week 3 as compared to week 6 ( $p=0.028)$ (Fig. 5).

Quantitative analysis of SHAM rats demonstrated increased BV from week 1 to week $6(p=0.048)$. In terms of BV/TV, an increase was observed in week 1 and 3 as compared to week $6(p=0.029$ and $p=0.016$, respectively). Tb.N was larger in week 1 as compared to week $3(p=0.025)$ and $6(p=0.021)$. Tb.Th increased between week 1 and 3 as compared to week 6 $(p=0.023$ and $p=0.006$, respectively). A decrease in 
$\mathrm{Tb} . S p$ was observed from week 1 to week $6(p=0.008)$ (Fig. 5).

Comparing OVX and SHAM groups, there was significantly larger TV at week 3 and $\mathrm{BV}$ at week 1 for the SHAM group ( $p=0.00$ and $p=0.00)$. Tb.N was also significantly larger at week 3 in the SHAM group $(p=0.00)$. Tb.Sp was larger in the OVX group at week $1(p=0.02)$. SHAM rats showed more ConnDens at week $3(p=0.01)$ and $\operatorname{BMD}(\mathrm{TV})$ at week $6(p=0.04)$ as compared to the OVX group. No significant differences were detected between groups in $\mathrm{BMD}(\mathrm{BV})$ and SMI (Fig. 5).

\section{Histomorphometric results}

Complete bony bridging at 6 weeks occurred for all metaphyseal-osteotomy rats. 3 stages were observed during healing (Fig. 6,7). Stage 1, with fibrous tissue consisting of disorganised orientation of collagen fibres and a collection of immune and mesenchymal cells. Stage 2, with a transitional zone consisting of maturing fibrous tissue and mesenchymal cells differentiating into bone cells, including osteoblasts and osteoclasts. In addition, early trabecular bone formation and disorganised woven bone were observed. Stage 3, with cortical bone ends mostly unified. The trabecular bone also underwent transformation to lamellar bone and further remodelling. Osteoblasts were observed to further differentiate into osteocytes, which were embedded into lamellar bone structures. At week 1, less fibrous tissue and more osseous tissue were observed for the SHAM group as compared to the OVX group. At week 3, the amount and staining intensity of the newly formed osseous tissue at the osteotomy site for the SHAM group provided evidence of more mature bone formed as compared to the OVX group. At week 6, the healing was more mature, with much more dense osseous tissue observed as compared to the OVX group.

Quantitatively, for the OVX group, osteoblast density increased from week $1(p=0.005)$ and 3 $(p=0.017)$ as compared to week 6. B.Ar/T.Ar increased a

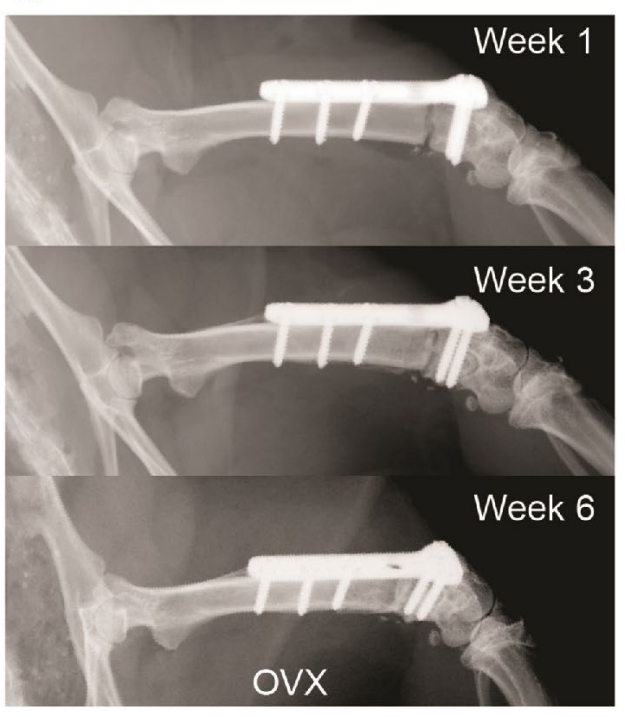

C
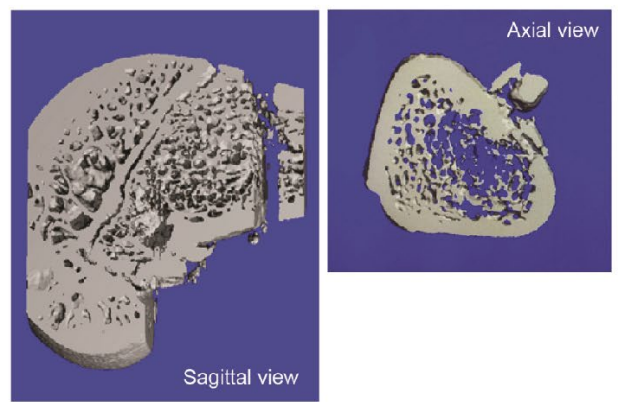

b

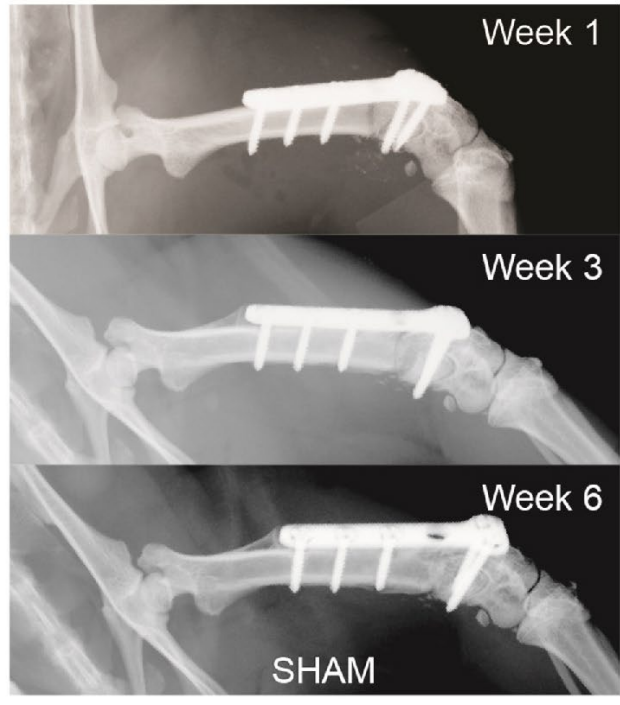

d
Week 3
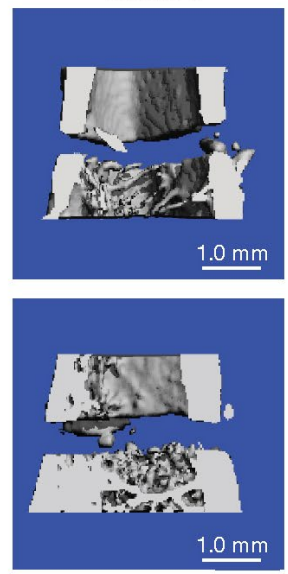

Week 6
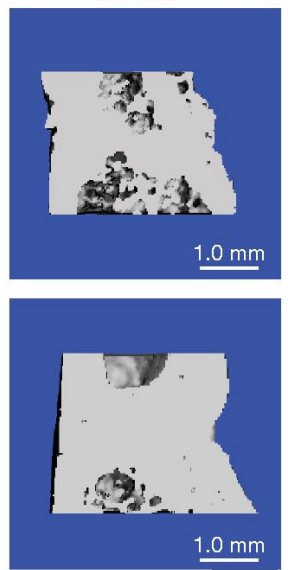

Fig. 3. Model establishment and monitoring of the healing process. (a) Serial X-ray images showing representative OVX samples. (b) Serial X-ray showing representative SHAM samples. (c) Three-dimensional (3D) reconstructed $\mu \mathrm{CT}$ images of the metaphyseal osteotomy site at week 1 , providing evidence of trabecular bone at the proximal and distal ends. (d) Serial representative 3D reconstructed images of the metaphyseal osteotomy site comparing OVX versus SHAM group. 
from week 1 to week $6(p=0.003)$. Osteoblast density was higher at week 1 and 3 for the SHAM group (151.9 \pm 41.4 and $156.5 \pm 48.5$ osteoblasts/ $\mathrm{mm}^{2}$, respectively) as compared to the OVX group ( $0 \pm 0$ and $50.6 \pm 21.1$ osteoblasts $/ \mathrm{mm}^{2}$, respectively $)$ ( $p=0.003$ and $p=0.026$, respectively). Osteoclast density was higher at week 1 for the SHAM group $\left(96.7 \pm 41.4\right.$ osteoclasts $\left./ \mathrm{mm}^{2}\right)$ as compared to the OVX group $\left(4.6 \pm 7.97\right.$ osteoclasts $\left./ \mathrm{mm}^{2}\right)(p=0.019)$. B.Ar/T. Ar was higher at week 1 for the SHAM group as compared to the OVX group ( $p=0.012$ ) (Fig. 5,8).

Qualitatively, trabecular bone was decreased after ovariectomy. At week 1, more fibrous tissue and less osseous tissue were observed in the OVX group. At week 3, more trabecular bone and newly formed osseous tissue were observed in the SHAM group. At week 6 , SHAM rats showed more mature and dense osseous tissue (Fig. 6,7).

Safranin O staining confirmed the presence of a minimal amount of proteoglycans in the metaphyseal model. Image analysis showed minimal uptake of Safranin $\mathrm{O}$ for the OVX group with $0 \pm 0 \%$ at week $1,0.01 \pm 0.01 \%$ at week 3 and $0.12 \pm 0.12 \%$ at week 6. For SHAM rats, Safranin O uptake was $0 \pm 0 \%$ at week $1,0.01 \pm 0.01 \%$ at week 3 and $0.12 \pm 0.12 \%$ at week 6 . Both groups significantly lacked cartilage formation (Fig. 7).

\section{Mechanical testing}

Energy to failure was significantly higher for the SHAM $(38.9 \pm 6.21 \mathrm{~N} \times \mathrm{mm})$ as compared to the OVX group (11.2 $\pm 3.23 \mathrm{~N} \times \mathrm{mm} ; p=0.03)$. Normalisation with intact SHAM bone was $38.6 \pm 8.8 \%$ and OVX bone $29.4 \pm 22.8 \%(p=0.53)$. Mean ultimate load showed no significant difference between the SHAM $(17.7 \pm 11.9 \mathrm{~N})$ and the OVX group $(9.17 \pm 1.02$; $p=0.42)$. Normalisation with intact SHAM bone was $66.3 \pm 18.4 \%$ and OVX bone $29.4 \pm 5.0 \%(p=0.00)$. Mean stiffness showed no significant difference between the SHAM $(11.35 \pm 8.1 \mathrm{~N} / \mathrm{mm})$ as compared to the OVX group $(3.87 \pm 1.25 ; p=0.33)$. Normalisation with intact SHAM bone was $63.8 \pm 13.4 \%$ and OVX bone $24.0 \pm 4.6 \%(p=0.00)$. No failure was observed

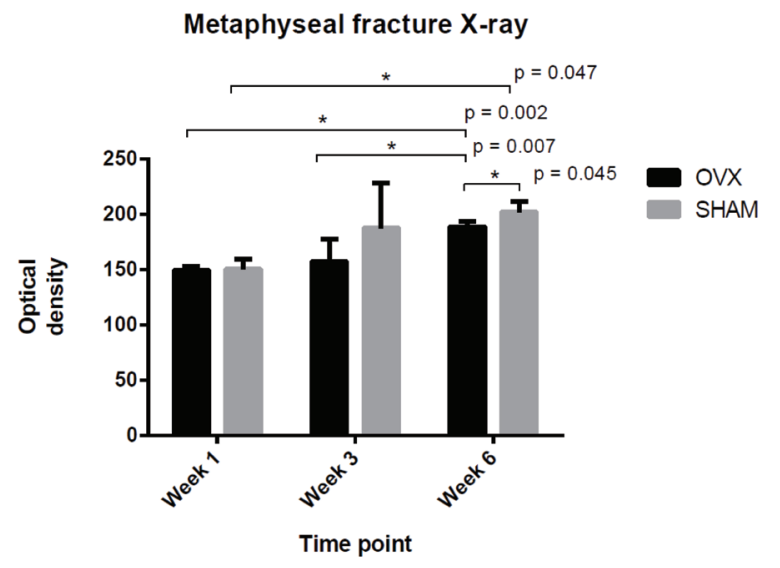

Fig. 4. Radiological evaluation of healing outcome in the metaphyseal osteotomy models. Optical density showing both OVX and SHAM groups. during mechanical testing, with all breakages at the healed osteotomy.

\section{Discussion}

The presented model satisfied the recent recommendations for the study of osteoporotic bone healing, with: i) induction of osteoporosis, ii) complete osteotomy unilaterally at the metaphyseal region, iii) internal fixation. These are essential qualities for a successful animal model to aid in clinical translation (Wong et al., 2018). Osteoporotic fracture healing should be concentrated in the metaphyseal region (Alt et al., 2013; Histing et al., 2012; Stuermer et al., 2010). More importantly, the key difference between the current metaphyseal model and the classic diaphyseal model was the presence of trabecular bone formation.

The ovariectomised rat model is Food and Drug Administration (FDA)-approved and is the most commonly used osteoporosis model for research (Egermann et al., 2005; Thompson et al., 1995). However, a limitation of ovariectomy models is that they cannot fully mimic the human situation (Egermann et al., 2005). Attempts with combined ovariectomy, calcium- and vitamin D-deficient diet and steroid therapy on sheep closely simulate human situations but have significant side-effects (Egermann et al., 2008). These sheep models have the benefit of a significant and reproducible reduction in cancellous BMD of more than $30 \%$. Furthermore, there are associated biomechanical properties and increased fracture risk (Egermann et al., 2008). Sheep models also have the benefits of sizable bones for fracture fixation. The rebound of BMD after steroid therapy allows a window for experimental studies (Goldhahn et al., 2005). Currently, only ovariectomised nonhuman primates closely resembles the clinical situation but are uncommonly used due to costs, ethical issues and handling problems (Egermann et al., 2005).

The traditional Bonnaren and Einhorn (1984) rat model provides a valuable guideline for the creation of a new animal model. The current model is a unique and refined version of the existing osteoporotic metaphyseal fracture models (Wong et al., 2018). The model is well suited for investigation of drugs and non-invasive interventions. On the other hand, the metaphyseal defect model by Alt et al. (2013) is best suited for studying biomaterials.

The clear difference in healing between SHAM and OVX rats showed the importance and potential of enhancing fracture healing for osteoporotic patients. Radiologically, X-ray radiopacity was significantly lower for the OVX group at week 6. $\mu \mathrm{CT}$ results also demonstrated less mature and decreased bone healing, with BV/TV significantly lower in the OVX group. Significantly lower BV and TV in the OVX group at week 1 showed the delay at early phases of osteoporotic bone healing. 
SHAM

VOX-BV

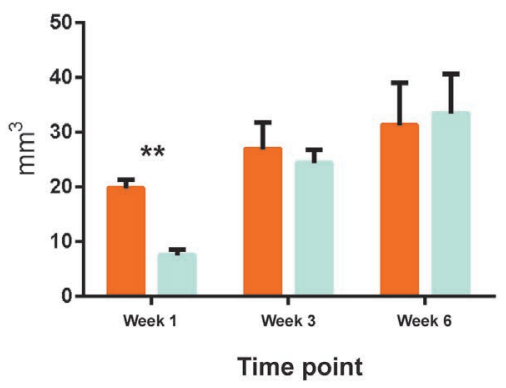

DT-Tb.N

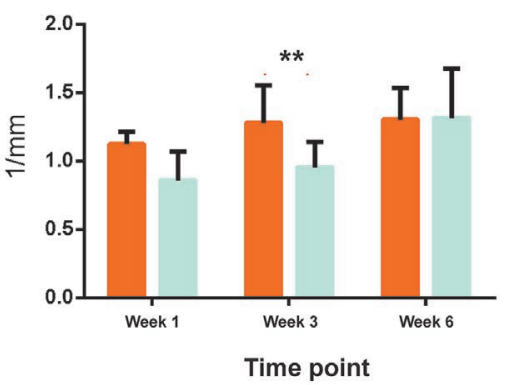

$B M D(T V)$

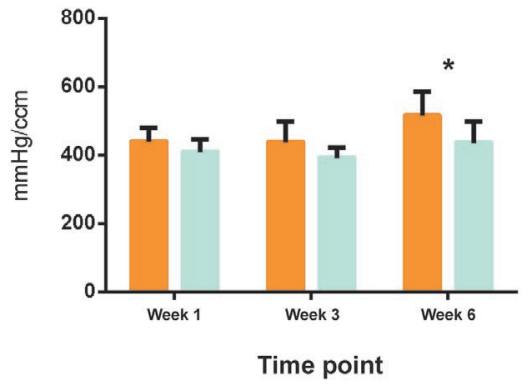

Conn-Dens

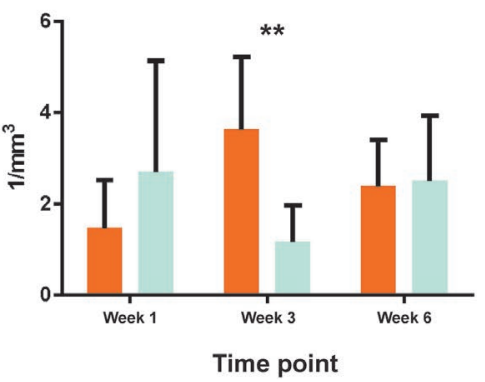

VOX-TV

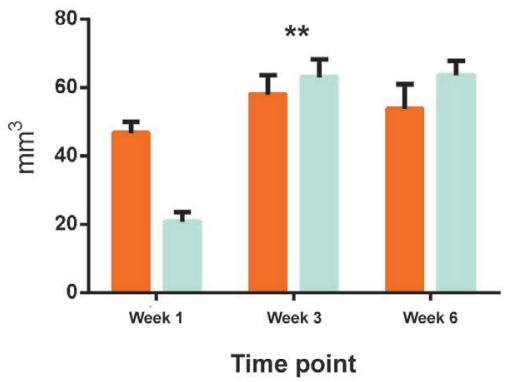

DT-Tb.Th

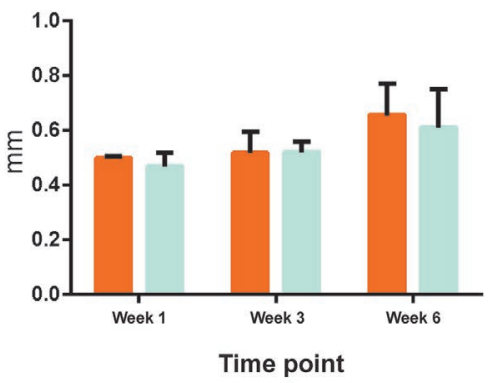

$\mathrm{BMD}(\mathrm{BV})$

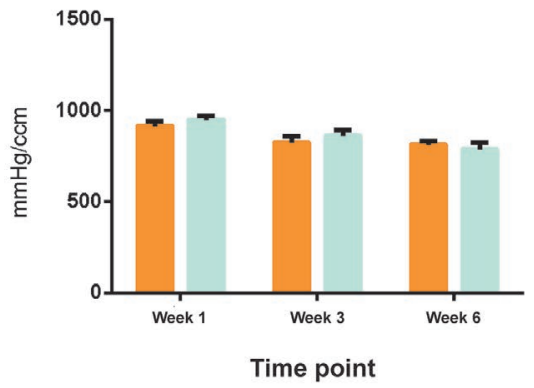

TRI-SMI

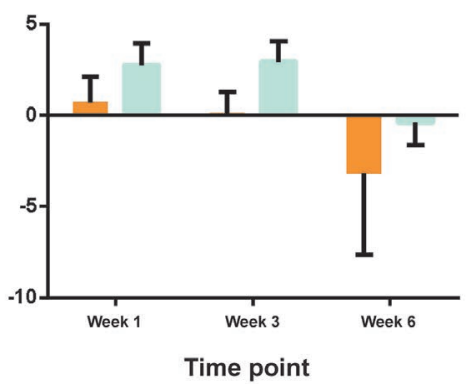

VOX-BV/TV

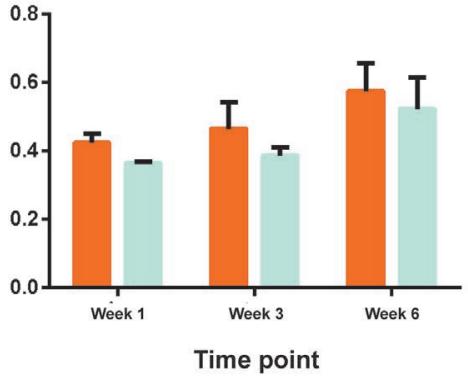

DT-Tb.Sp

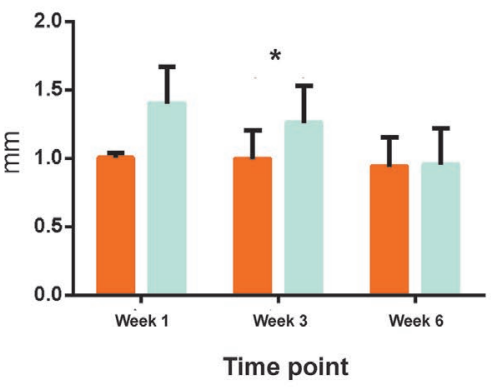

Fig. 5. MCT and histomorphometry results of OVX and SHAM groups. Comparing OVX and SHAM groups, there was significantly larger TV at week 3 and BV at week 1 for the SHAM group $(p<0.001$ and $p<0.001)$. Tb.N was also significantly larger at week 3 in the SHAM group $(p<0.001)$. Tb.Sp was larger for the OVX group at week $1(p=0.02)$. SHAM rats showed higher Conn-Dens at week $3(p=0.01)$ and higher $\operatorname{BMD}(\mathrm{TV})$ at week $6(p=0.04)$ as compared to OVX rats. ${ }^{*} p<0.05,{ }^{* *} p<0.01$. 


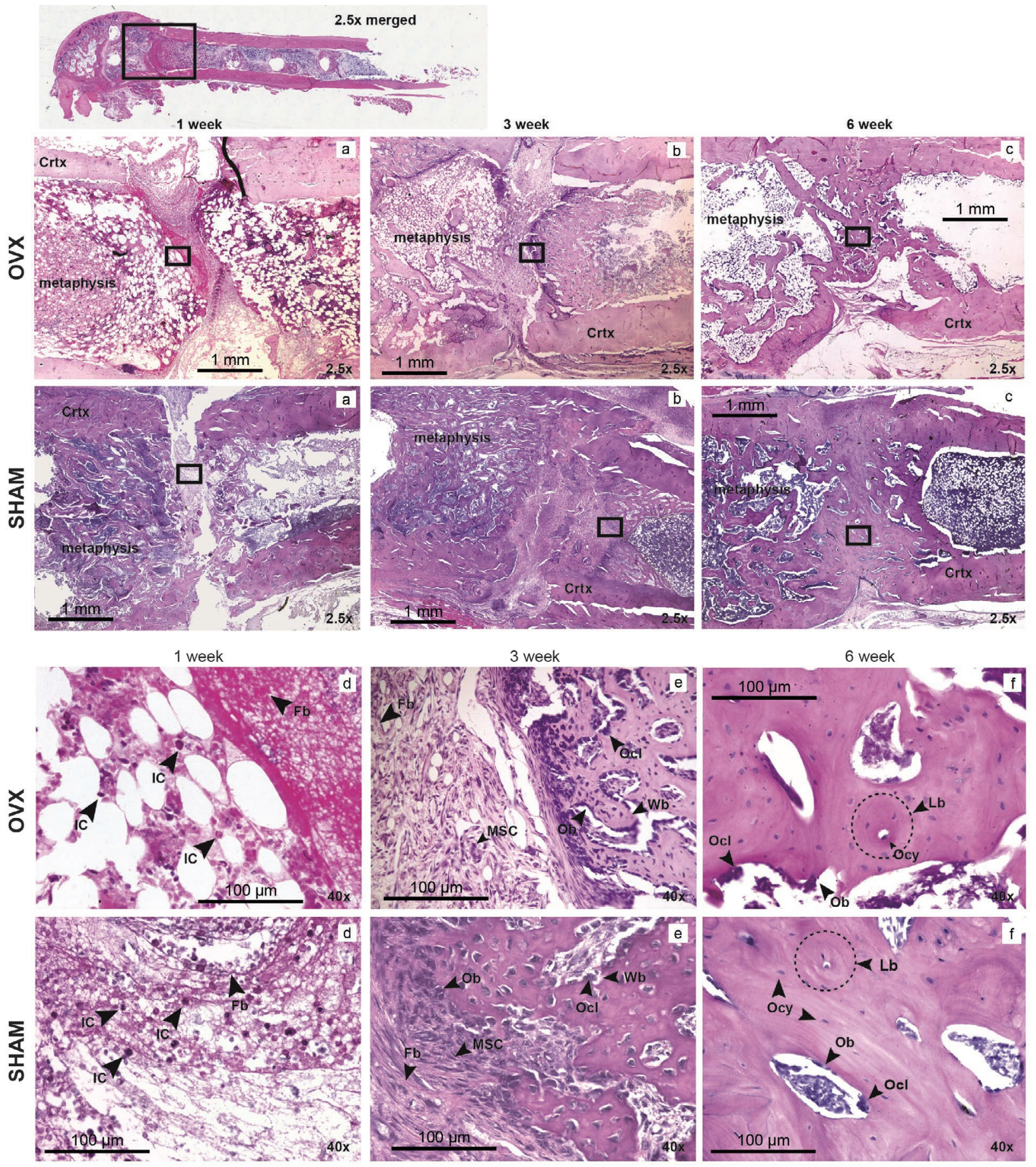

Fig. 6. Histology at the metaphyseal fracture site in SHAM and OVX rats. (a-c) Serial H\&E histology at 2.5× magnification showing SHAM versus OVX group fracture site from week 1 to week 6 post-fracture induction, followed by (d-f) histology at 40× magnification. (a,d) At week 1 post-fracture, accumulation of round-shaped immune cells (IC) of various sizes could be observed, with apparent laying down of ground substances and the formation of fibrous tissue $(\mathrm{Fb})$. Less fibrous tissue and more osseous tissue were observed in the SHAM group as compared to the OVX group. $(\mathbf{b}, \mathbf{e})$ The amount of trabeculae increased at week 3 post-fracture; a clear transition zone could be observed consisting of various cell types, demonstrating actively differentiating mesenchymal cells (MSC) surrounded by slowly maturing (pink to purple shifting) fibrous tissue. The amount and stain intensity of the newly formed osseous tissue in the SHAM group provided evidence of more matured bone formed at this time point as compared to the OVX group. Osteoblast $(\mathrm{Ob})$ and osteoclast $(\mathrm{Ocl})$ were both observed toward the woven bone structures in the endosteal space. Chondrocyte and traces of endochondral ossification could not be observed. (c,f) At week 6 post-fracture, newly formed bone further mineralised to form lamellar bone (Lb) and remodelling continued, as indicated by terminally differentiated osteocytes (Ocy). Healing in the SHAM group was more advanced, with osseous tissue much more dense as compared to the OVX group. 
Histomorphometric results concurred with radiological data. Less osseous tissue formed in the OVX group at week 1, with significantly less bone area ratio and osteoblasts. Osteoblasts differentiate from mesenchymal stem cells at early stages of fracture healing (Ghiasi et al., 2017). At week 3, the OVX group continued to lag behind in newly formed bone, osteoblast and osteoclast number. Furthermore, the decrease in osteoclasts at week 6 indicated that the SHAM group healed faster, as shown in a previous study (Schindeler et al., 2008).

Mechanical results revealed that energy to failure was weaker after bone union in the OVX group. Although ultimate force and stiffness were not significant, the trend showed average values to be lower in the OVX group. The overall strength was decreased in osteoporotic bone healing. Normalisation for mean ultimate load and stiffness was significantly less in the OVX group. The trend could also be observed in the mean ultimate failure. These results not only implied a lower biomechanical strength for healed osteoporotic bone as compared to normal bone, but also to the contralateral intact bone. This reinforced the evidence of a weaker and poor healing of osteoporotic fractures.

Many existing clinical and basic science studies have shown that osteoporotic fracture healing is delayed as compared to normal bone (Cheung et al., 2016). Convincing evidence exist that biomechanical properties and mineralisation are impaired when compared to normal bone (Chen et al., 2016). In fact, the impairment of osteoporotic fracture healing exists from the early to late phases of fracture healing. In the early phase, mesenchymal stem cell recruitment (Wei et al., 2016) and local fracture site inflammatory response are impaired (Chow et al., Vibration treatment modulates macrophage polarization and enhances early inflammatory response in estrogendeficient osteoporotic fracture healing; paper submitted for publication). Callus formation (Shi et al., 2010) and angiogenesis (Cheung et al., 2012) are also decreased during the callus phase. During the
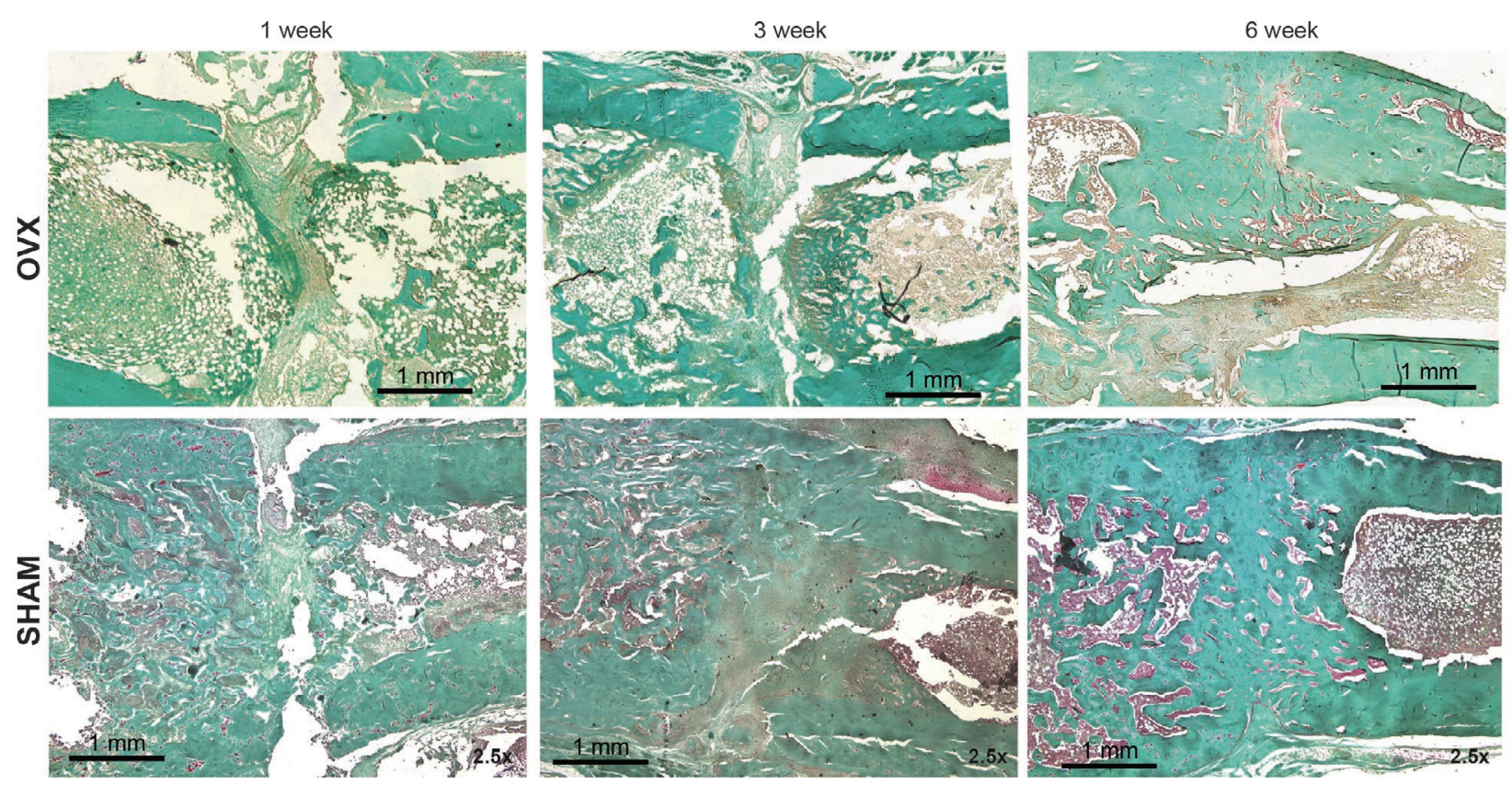

Fig. 7. Representative histology of Safranin $O$ with fast green counter-staining demonstrated the lack of endochondral bone healing.

Ob density

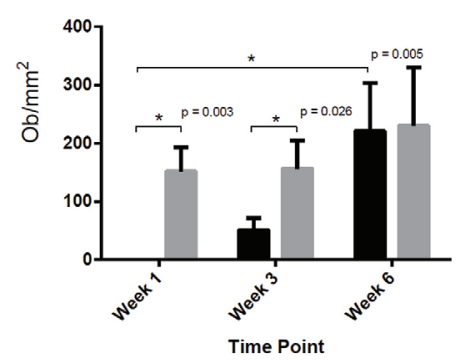

Oc density

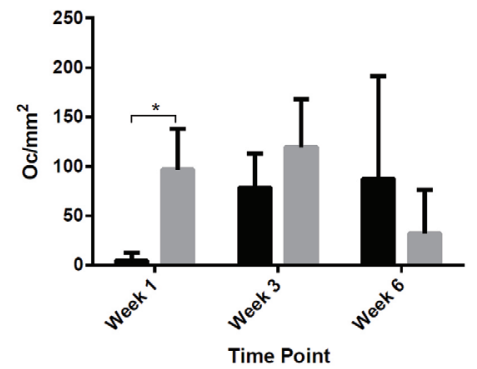

Bone area ratio

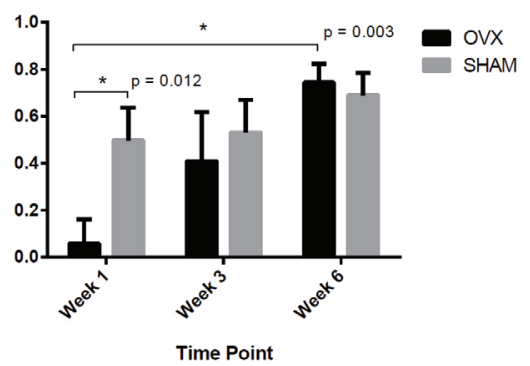

Fig. 8. Histomorphometric results for OVX and SHAM groups. (a) Osteoblast density, (b) osteoclast density and (c) bone area ratio. 
late phase, the capacity of remodelling is also delayed (Chow et al., 2011). During the osteoporotic fracture healing, expression of chondrogenesis, osteogenesis and remodelling genes is decreased (Chung et al., 2014). More importantly, biomechanical strength of ovariectomy-induced osteoporotic rats is reduced as compared to age-matched non-osteoporotic bone, when the fracture heals (Shi et al., 2010). Bone healing in osteoporotic patients is also delayed; the alterations characterised by poor bone quality (Tarantino et al., 2011). These findings also concur with the results observed in the present study, where the OVX group had less mature healing as compared to the SHAM group. As a result, poor functional recovery, pain and fixation failure most often occurs in elderly patients (Wong et al., 2018). Therefore, the enhancement of osteoporotic fracture healing is a major goal in modern fracture management.

Currently, the most feasible method for simulating a metaphyseal fracture in a small animal is an osteotomy with plate fixation (Cheung et al., 2016; Wong et al., 2018). The use of a closed method for a consistent metaphyseal fracture with internal fixation is beyond current technology. A guillotine to fracture the metaphyseal region would lead to comminution and irreproducibility.

The primary objective of creating an osteoporotic metaphyseal osteotomy model fixed with plate and screws was met. Future pre-clinical studies and interventions should select a relevant model before proceeding to clinical trials.

\section{Conclusion}

The presented model had the essential quality of a successful animal model to aid in the clinical translation of future studies for osteoporotic metaphyseal healing. It also could serve as a platform for other potential interventions.

\section{Acknowledgements}

The project was supported by the Health and Medical Research Fund (HMRF), Food and Health Bureau, Government of the Hong Kong Special Administrative Region (Ref: 04152406), Asian Association for Dynamic Osteosynthesis (AADO) Research Fund (Ref: AADO-RF2016-2Y), Germany/ Hong Kong Joint Research Scheme, RGC (Ref: G-CUHK401/15) and Theme-based Research Scheme (Ref: T13-402/17-N). Sources played no role in experiments and analysis.

\section{References}

Alt V, Thormann U, Ray S, Zahner D, Durselen L, Lips K, El Khassawna T, Heiss C, Riedrich A,
Schlewitz G, Ignatius A, Kampschulte M, von Dewitz H, Heinemann S, Schnettler R, Langheinrich A (2013) A new metaphyseal bone defect model in osteoporotic rats to study biomaterials for the enhancement of bone healing in osteoporotic fractures. Acta Biomater 9: 7035-7042.

Auer JA, Goodship A, Arnoczky S, Pearce S, Price J, Claes L, Von Rechenberg B, Hofmann-Amtenbrinck M, Schneider E, Muller-Terpitz R, Thiele F, Rippe KP, Grainger DW (2007) Refining animal models in fracture research: seeking consensus in optimising both animal welfare and scientific validity for appropriate biomedical use. BMC Musculoskelet Disord 8: 72. DOI: 10.1186/1471-2474-8-72.

Bindl R, Oheim R, Pogoda P, Beil FT, Gruchenberg K, Reitmaier S, Wehner T, Calcia E, Radermacher P, Claes L, Amling M, Ignatius A (2013) Metaphyseal fracture healing in a sheep model of low turnover osteoporosis induced by hypothalamic-pituitary disconnection (HPD). J Orthop Res 31: 1851-1857.

Bonnarens F, Einhorn TA (1984) Production of a standard closed fracture in laboratory animal bone. J Orthop Res 2: 97-101.

Chen L, Yang L, Yao M, Cui XJ, Xue CC, Wang YJ, Shu B (2016) Biomechanical characteristics of osteoporotic fracture healing in ovariectomized rats: a systematic review. PLoS One 11: e0153120. DOI: 10.1371/journal.pone.0153120.

Cheung WH, Miclau T, Chow SK, Yang FF, Alt V (2016) Fracture healing in osteoporotic bone. Injury 47 Suppl 2: S21-26.

Cheung WH, Sun MH, Zheng YP, Chu WC, Leung AH, Qin L, Wei FY, Leung KS (2012) Stimulated angiogenesis for fracture healing augmented by low-magnitude, high-frequency vibration in a rat model-evaluation of pulsed-wave doppler, 3-D power Doppler ultrasonography and micro-CT microangiography. Ultrasound Med Biol 38: 2120 2129.

Chow DH, Leung KS, Qin L, Leung AH, Cheung WH (2011) Low-magnitude high-frequency vibration (LMHFV) enhances bone remodeling in osteoporotic rat femoral fracture healing. J Orthop Res 29: 746-752.

Chung SL, Leung KS, Cheung WH (2014) Lowmagnitude high-frequency vibration enhances gene expression related to callus formation, mineralization and remodeling during osteoporotic fracture healing in rats. J Orthop Res 32: 1572-1579.

Egermann M, Goldhahn J, Holz R, Schneider E, Lill CA (2008) A sheep model for fracture treatment in osteoporosis: benefits of the model versus animal welfare. Lab Anim 42: 453-464.

Egermann M, Goldhahn J, Schneider E (2005) Animal models for fracture treatment in osteoporosis. Osteoporos Int 16 Suppl 2: S129-138.

Ghiasi MS, Chen J, Vaziri A, Rodriguez EK, Nazarian A (2017) Bone fracture healing in mechanobiological modeling: a review of principles and methods. Bone Rep 6: 87-100.

Goldhahn J, Jenet A, Schneider E, Lill CA (2005) Slow rebound of cancellous bone after mainly steroid- 
induced osteoporosis in ovariectomized sheep. J Orthop Trauma 19: 23-28.

Histing T, Klein M, Stieger A, Stenger D, Steck R, Matthys R, Holstein JH, Garcia P, Pohlemann T, Menger MD (2012) A new model to analyze metaphyseal bone healing in mice. J Surg Res 178: 715-721.

Kolios L, Hoerster AK, Sehmisch S, Malcherek MC, Rack T, Tezval M, Seidlova-Wuttke D, Wuttke W, Stuermer KM, Stuermer EK (2010) Do estrogen and alendronate improve metaphyseal fracture healing when applied as osteoporosis prophylaxis? Calcif Tissue Int 86: 23-32.

Komrakova M, Weidemann A, Dullin C, Ebert J, Tezval M, Stuermer KM, Sehmisch S (2015) The impact of strontium ranelate on metaphyseal bone healing in ovariectomized rats. Calcif Tissue Int 97: 391-401.

Larsson S (2002) Treatment of osteoporotic fractures. Scand J Surg 91: 140-146.

Leung KS, Shi HF, Cheung WH, Qin L, Ng WK, Tam KF, Tang N (2009) Low-magnitude highfrequency vibration accelerates callus formation, mineralization, and fracture healing in rats. J Orthop Res 27: 458-465.

Leung KS, Siu WS, Li SF, Qin L, Cheung WH, Tam KF, Lui PP (2006) An in vitro optimized injectable calcium phosphate cement for augmenting screw fixation in osteopenic goats. J Biomed Mater Res B Appl Biomater 78: 153-160.

Rachner TD, Khosla S, Hofbauer LC (2011) Osteoporosis: now and the future. Lancet 377: 12761287.

Schindeler A, McDonald MM, Bokko P, Little DG (2008) Bone remodeling during fracture repair: The cellular picture. Semin Cell Dev Biol 19: 459-466.

Shi HF, Cheung WH, Qin L, Leung AH, Leung KS (2010) Low-magnitude high-frequency vibration treatment augments fracture healing in ovariectomyinduced osteoporotic bone. Bone 46: 1299-1305.

Solomon DH, Patrick AR, Schousboe J, Losina E (2014) The potential economic benefits of improved postfracture care: a cost-effectiveness analysis of a fracture liaison service in the US health-care system. J Bone Miner Res 29: 1667-1674.

Stuermer EK, Sehmisch S, Daub F, Komrakova M, Tezval M, Stuermer KM (2013) Raloxifene supports early fracture healing more than estrogen in ovariectomized rats. Osteologie 22: 290-297.
Stuermer EK, Sehmisch S, Rack T, Wenda E, Seidlova-Wuttke D, Tezval M, Wuttke W, Frosch KH, Stuermer KM (2010) Estrogen and raloxifene improve metaphyseal fracture healing in the early phase of osteoporosis. A new fracture-healing model at the tibia in rat. Langenbecks Arch Surg 395: 163-172.

Tarantino U, Cerocchi I, Scialdoni A, Saturnino L, Feola M, Celi M, Liuni FM, Iolascon G, Gasbarra E (2011) Bone healing and osteoporosis. Aging Clin Exp Res 23: 62-64.

Thompson DD, Simmons HA, Pirie CM, Ke HZ (1995) FDA guidelines and animal models for osteoporosis. Bone 17: 125S-133S.

Wei FY, Chow SK, Leung KS, Qin J, Guo A, Yu OL, Li G, Cheung WH (2016) Low-magnitude highfrequency vibration enhanced mesenchymal stem cell recruitment in osteoporotic fracture healing through the SDF-1/CXCR4 pathway. Eur Cell Mater 31: 341354.

Wong RMY, Choy MHV, Li MCM, Leung KS, S KHC, Cheung WH, Cheng JCY (2018) A systematic review of current osteoporotic metaphyseal fracture animal models. Bone Joint Res 7: 6-11.

\section{Web References}

1.https://www.mpi.govt.nz/dmsdocument/33585good-practice-guide-for-the-use-of-animals-inresearch-testing-and-teaching [10-05-2019].

\section{Discussion with Reviewers}

Stephan Zeiter: How technically challenging is this model?

Authors: The establishment of the animal model was not difficult but limited by the requirement of a precision bone-cutting instrument and an experienced small-animal surgeon. The surgery, involving the fixation of a mini-plate followed by the osteotomy at the metaphysis of the distal femur, was also not difficult to master and could easily be made highly repeatable with some practice. Working with a surgical assistant would greatly help the process.

Editor's note: The Scientific Editor responsible for this paper was Juerg Gasser. 